\title{
A Dynamic and Scalable User-centric Route Planning Algorithm Based on Polychromatic Sets Theory
}

\author{
Peisong Li, Xinheng Wang, Senior Member, IEEE, Honghao Gao, Senior Member, IEEE, Xiaolong Xu, \\ Muddesar Iqbal, and Keshav Dahal, Senior Member, IEEE
}

\begin{abstract}
Existing navigation services provide route options based on a single metric without considering user's preference. This results in the planned route not meeting the actual needs of users. In this paper, a personalized route planning algorithm is proposed, which can provide users with a route that meets their requirements. Based on the multiple properties of the road, the Polychromatic Sets (PS) theory is introduced into route planning. Firstly, a road properties description scheme based on the PS theory was proposed. By using this scheme, users' travel preferences can be quantified, and then personalized property combination schemes can be constructed according to these properties. Secondly, the idea of setting priority for road segments was utilized. Based on a user's travel preference, all the property combination schemes can be prioritized at relevant levels. Finally, based on the priority level, an efficient path planning scheme was proposed, in which priority is given to the highest road segments in the target direction. In addition, the system can constantly obtain real-time road information through mobile terminals, update road properties, and provide other users with more accurate road information and navigation services, so as to avoid crowded road segments without excessively increasing time consumption. Experiment results show that our algorithm can realize personalized route planning services without significantly increasing the travel time and distance. In addition, source code of the algorithm has been uploaded on GitHub for this algorithm to be used by other researchers.
\end{abstract}

Index Terms-User-centric, Route planning, Polychromatic Sets Theory.

\section{INTRODUCTION}

Manuscript received November 21, 2020; revised March 3, 2021; accepted May 21, 2021. The work was supported in part by the XJTLU Research Development Funding + RDF-19-01-14, XJTLU Key Programme Special Fund KSF-E-64, and the National Natural Science Foundation of China under Grant 62072255. Corresponding authors: Xinheng Wang and Honghao Gao)

P. Li and X. H. Wang are with the School of Advanced Technology, Xi' an Jiaotong-Liverpool University, Suzhou 215123, China (E-mail: peisong.li20@student.xjtlu.edu.cn, xinheng.wang@xjtlu.edu.cn).

H. Gao is with the School of Computer Engineering and Science, Shanghai University, Shanghai 200444, China (E-mail: gaohonghao@ shu.edu.cn).

$\mathrm{X}$. Xu is with the Jiangsu Key Laboratory of Big Data Security and Intelligent Processing, Nanjing University of Posts and Telecommunications, Nanjing 210023, China (E-mail: xuxl@njupt.edu.cn).

M. Iqbal is with the Division of Computer Science and Informatics, School of Engineering at London South Bank University, London, UK (Email: m.iqbal@1sbu.ac.uk).

K. Dahal is with the AVCN research centre at the School of Computing, Engineering and Physical Sciences, University of the West of Scotland, Paisley, Scotland, UK (E-mail: keshav.dahal@uws.ac.uk).
$\mathbf{R}$ OUTE planning is one of the most basic functions of navigation systems, which can help travelers determine the best route that meets the requirement [1]-[3]. Existing navigation applications such as Google Maps and Waze can provide accurate services with options, for example, multiple routes are provided for selection on Google Maps before departure and accurate arrival time could be assured by Waze owing to the update of real-time traffic conditions. Some other services such as going to the petrol station in the middle of the journey are also considered, which makes the services more personalized.

Normally, these services rely heavily on one or two metrics such as the shortest distance or the shortest travel time. However, users' demands are diverse. Some drivers might desire to the destination by using the least time, so highways or street roads with less traffic are selected, but they have to drive very fast or make a lot of right or left turns. Others might prefer to drive an easy way, such as driving on a more straightforward road despite of the heavy traffic or not. Upon existing navigation systems, it is obvious that recommending the same path for all the users based on predefined metric(s) cannot meet the individual needs for different users. In this case, the recommended route is usually not selected [4]. However, there are no other choices available in existing navigation applications. In addition, according to the study in [5], the routes that are usually selected by the drivers are significantly longer than their shortest route when the route selection criterion is either 'travel length' or 'travel time'. Therefore, authors declared that "... algorithms based on shortest route to represent routes may not capture real-world route choice decisions.", which means that routes computed based on a single-criterion shortest route algorithm like Dijkstra may not be desirable for a driver.

Recently, in order to solve these problems, multi-criterion route planning has become a research hot-spot for public travel information services [6]-[8]. These methods consider multiple criteria and weight each criterion to make an optimal new choice. However, in reality, it is still a onecriterion navigation method. It cannot offer multiple choices to the users, particularly to those during the navigation. In addition, these methods don't have the scalability in terms of metrics. The number of metrics is limited. When a metric 
needs to be considered into the service, a new algorithm has to be designed to meet the demand.

We argue the reason behind this is because there lacks of a systematic mathematical model to describe the conditions or properties of the traffic and then make recommendations based on these conditions. In this paper, we have proposed a new property description method based on Polychromatic Sets (PS) Theory, where unlimited number of metrics can be described and used for forming new routes. This makes the system scalable, and the selection of routes can be based on user's preferences, rather than one or two physical parameters.

In this new proposed method, firstly, various features and conditions of the road and traffic, such as expressway, serious congestion, toll road and so so, where a term 'property' is used to refer to these features and conditions, can be described comprehensively and combinations of route properties can be established according to different travel plans. Then, according to the specific travel plan, priority is assigned to the properties. In the route planning stage, the high-priority road segment is selected first, and then lowpriority road segments are selected next, if no high-priority segment is available. In addition, priority should be given to the road segment in the direction to the destination, which can effectively reduce the search area and ensure that the planned path is close to the shortest path. In this way the execution efficiency of the algorithm could be improved.

The contributions of this paper are summarised in the following three aspects:

(1) A road property description scheme based on PS theory is provided. This scheme is capable of including main properties of road networks in route planning.

(2) A user-centric road priority setting method is proposed. Under different and personalized travel plans, the priority of each road segment can be dynamically adjusted and selected to plan the route.

(3) An efficient route planning process is proposed. When carrying out route planning, the road segments with high priority and in the direction to the destination will be chosen first, which can ensure that the planned route not only meets the user's preferences and the specific travel plan, but also effectively reduces the search area and further saving time used for route planning.

The remainder of this paper is organized as follows: In Section II, related work is reviewed. It also demonstrates how our work is different from existing research. In Section III, a framework of a property description method and route planning process is proposed. The experimental setup and the performance evaluation results are presented in Section IV to verify the effectiveness of the proposed approach. Finally, this paper is concluded and the future research directions are discussed in Section V.

\section{RELATED WORK}

Traditional travel route planning provides a route between an origin and a destination according to a certain metric, such as the shortest distance or the shortest travelling time. Dijkstra's algorithm [9] is the most well-known method for solving the shortest path problem. However, Dijkstra's algorithm needs to be modified for some real travel applications, due to its inefficiency. To address this problem, Delling et al. [10] proposed a customizable route planning for real-time queries in terms of arbitrary metrics, including avoidance of U-turns and/or left turns.

Traditional travel route planning schemes have other two limitations. First, some factors, such as road safety and traffic jams, are usually latent in travelling and difficult to access; hence, traditional route planning only considers limited factors and cannot make a comprehensive consideration of travel. Second, traditional route planning algorithms are not personalized because they do not consider user's travel preferences and provide only generic recommendations to the public.

User-centric is an increasingly concerned direction, and the future development will definitely be based on user experience. Several previous studies have investigated usercentric route planning algorithms. Table I shows the details of the studies. In [11], users are allowed to decide the bus route. A geospatial decision support tool for bus travel planning based on design and modeling considerations was developed and implemented in this paper, which is more individual-centric than existing approaches. The distinctive characteristic of the proposed tool is the incorporation of bus riders' cognition, information processing, and decisionmaking process during their search for an appropriate bus transit travel plan. Authors in [12] proposed a personalized route planning query method. They developed new preprocessing schemes that allow for real-time personalized route planning in huge road networks while keeping the memory footprint of the preprocessed data and subsequent queries small. Researchers in [13] defined users' travel behaviors from their historical GPS trajectories and proposed two personalized travel route recommendation methods collaborative travel route recommendation (CTRR) and an extended version of CTRR (CTRR+). In [14], a probabilistic driving preference model in multi-attribute dynamic road traffic network was proposed, which can be used to solve personalized Skyline path.

Nadi and Delavar [15] proposed a model for the personalized multi-criterion route planning that fused a pair-wise comparison method and quantifier-guided ordered weighted averaging (OWA) aggregation operators to determine an impedance value for each link of the urban transportation network. Sadeghi Niaraki and Kim [16] integrated an Analytic Hierarchical Process (AHP) with a generic ontologybased architecture to identify and define a class of road segment criteria for personalized route planning. In the two mentioned studies, the personalized multi-criterion route planning problem has been simplified to a single objective optimization routing problem. When they consider a certain index, the weight of this index increases, but they ignore other indices and reduce the weight of other indices. As a 
TABLE I

SUMMARY OF RELATED WORK

\begin{tabular}{|c|c|c|c|c|c|c|c|}
\hline \multirow{2}{*}{ Related works } & \multicolumn{7}{|c|}{ Key features } \\
\hline & User-centric & Multi-criteria & Optimized route planning process & Real-time update & Shortest Distance & Scalability & Historical data \\
\hline Proposed Polychromatic Sets method & $\checkmark$ & $\checkmark$ & $\checkmark$ & $\checkmark$ & $\checkmark$ & $\checkmark$ & \\
\hline Graph partitioning [8] & $\checkmark$ & & $\checkmark$ & $\checkmark$ & & & \\
\hline Behavioral information processing [9] & $\checkmark$ & & & & & & \\
\hline Pruning algorithms [10] & $\checkmark$ & & & $\checkmark$ & $\checkmark$ & & \\
\hline Collaborative filtering [11] & $\checkmark$ & & & & & & $\checkmark$ \\
\hline Context-aware routing [12] & $\checkmark$ & & & & & & $\checkmark$ \\
\hline Ordered weighted averaging [13] & $\checkmark$ & $\checkmark$ & & & & & \\
\hline Analytic Hierarchical Process [14] & $\checkmark$ & $\checkmark$ & & & & & \\
\hline Intelligent personalized route assistant [15] & $\checkmark$ & $\checkmark$ & & & & & \\
\hline Road qualities and driver profiles based model [16] & $\checkmark$ & $\checkmark$ & & $\checkmark$ & & & \\
\hline Crowdsourcing and digital footprints [17] & $\checkmark$ & & & $\checkmark$ & & & $\checkmark$ \\
\hline Time-Dependent Customizable Route Planning [18] & $\checkmark$ & & & $\checkmark$ & & & $\checkmark$ \\
\hline Personalized fuel consumption model [19] & $\checkmark$ & & & $\checkmark$ & & & $\checkmark$ \\
\hline
\end{tabular}

result, the planned route does not meet the expectation of users and an "abnormal" route is provided.

Some researchers are focusing on how to provide route navigation options based on the road information and driver's profiles. Marina et al. [17] focused on the personalized routes problem and then presented a specific case of designing accessible and green pedestrian routes. Similarly, a route planning framework was proposed in [18], which utilizes the in-vehicle and smartphone sensors to build a crowdsensed database on segment quality and the driver's personalized behavioral information in different driving environments. A two-phase approach for personalized trip planning was proposed in [19]. In the route search phase, TRIPPLANNER works interactively with users to generate candidate routes with specified venues; in the route augmentation phase, TRIPPLANNER applies heuristic algorithms to add user's preferred venues iteratively to the candidate routes, with the objective of maximizing the route score while satisfying both the venue visiting time and total travel time constraints.

Some researchers focus on the impact of real-time road traffic information on route planning. The dynamic and time-dependent route planning problem was studied in [20], which takes both prediction (based on historical data) and live traffic into account. Traffic jams at different times of the day is also considered. A novel fuel-efficient pathplanning framework called Green Planner was presented in [21], which contains two phases. In the first phase, a Personalized Fuel Consumption Model (PFCM) for each driver was built, based on the individual driving behaviors and the physical features such as traffic lights, stop signs, and road network topology along the routes. In the second phase, the real-time traffic information was collected via a mobile crowdsensing manner. This method could estimate and compare the fuel cost among different routes for a given driver, and recommend him/her with the most fuel-efficient one.

However, above researchers didn't consider the multiproperty of the dynamic and complex road networks in reality. Some existing algorithms can analyze users' travel preferences according to their historical trajectories, but they cannot deal with users' new travel preferences. At the same time, the existing user-centric route planning algorithms can satisfy the user's single travel preference, but cannot solve the multi-objective travel requirements. In addition, towards the road property, the existing algorithms only focus on the traffic information, but not consider the road inherent properties sufficiently. Moreover, the calculation process of the algorithm is complex, so it is difficult to widely implement and deploy. Therefore, this paper aims to solve the problems that the planned route is not consistent with the user's current travel preference, so as to make the planned route more personalized. The route planning algorithm based on PS theory proposed in this paper fully considers the multiple properties of the road, and the travel plan can include multiple independent criteria, such as time, type of vehicle, road choice, and traffic conditions. This not only meets the user's multiple needs for the travel route, but also ensures that the planned route is as close as possible to the shortest route between two points.

\section{Polychromatic Sets Theory and Algorithm DESIGN}

A pioneering work in applying PS theory in computer networks was introduced in [22]. In that paper, the theory of the polychromatic sets and the way how the network properties were described by the theory was fully explained. Following the same principle of applying PS theory in computer networks, the properties of road networks can be described by this theory. An example of the road properties is listed in Table II.

\section{A. Road property description based on PS}

There are three important definitions in the PS-based road property description: Segment, Property and Property category.

1) Segment: Set $A$ is defined as a set of all the road segments in the road network:

$$
A=\left\{a_{1}, a_{2}, \cdots, a_{i}, \cdots, a_{n}\right\}
$$

where $a_{i}$ is an element of set $A$ and represents one road segment, $n$ is the total number of segments. 
2) Property (color): As mentioned earlier, road network has different features and conditions. In PS theory, the way to describe these features and conditions is called "property". In this case, one segment in the road network has a variety of properties. Examples of road properties are shown in Table II.

PS theory defines a property set by $F$, where for any segment $a_{i}$, the properties of $a_{i}$ is denoted as:

$$
F\left(a_{i}\right)=\left\{f_{1}, f_{2}, \cdots, f_{i}, \cdots, f_{q}\right\}
$$

where $f_{i}$ is an element of set $F\left(a_{i}\right)$ and represents one property, $q$ represents the total number of properties of road segment $a_{i}$. Unlike traditional set theories, set element is only one value. Here set element $a_{i}$ may have multiple properties and, thus, multiple values. This is the main difference between traditional set theories and PS theory. The detailed description of the PS theory can be found in [22].

Also, $F(A)$ is defined as the property set of all the segments in the road network:

$$
F(A)=\bigcup_{i=1}^{n} F\left(a_{i}\right)=\left\{f_{1}, f_{2}, \cdots, f_{i}, \cdots, f_{m}\right\}
$$

Therefore, the overall road network's properties can be calculated by a Cartesian product of $A$ and $F(A)$, shown in Eq. (4).

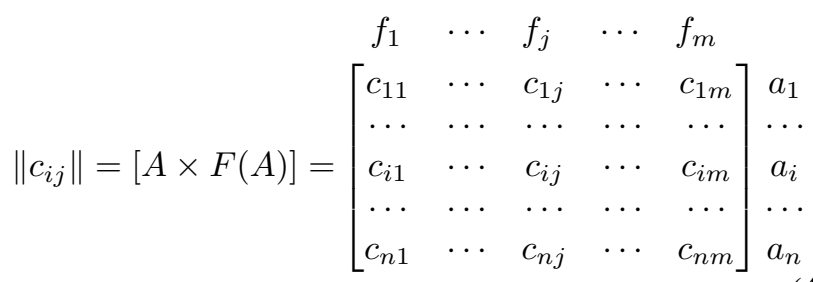

$[A \times F(A)]$ is a two-dimensional matrix, the size of it is $[n \times m]$. If one road segment $a_{i}$ is coloured as $f_{j}$, which means that $a_{i}$ has the property $f_{j}$, then the element $c_{i j}$ in the above matrix is set as 1 , otherwise, it is 0 .

3) Property category: Based on different standards of classification, the properties can be divided into different categories. For example, as shown in Table II, all these properties belong to more than 5 categories, including Road type, Speed level, Congestion, Road function, Toll status and so on.

$$
G(F)=\left[\begin{array}{c}
F_{1} \\
F_{2} \\
F_{3} \\
F_{4} \\
F_{5} \\
\cdots \\
F_{q}
\end{array}\right]=\left[\begin{array}{ccccc}
f_{1-1} & f_{1-2} & f_{1-3} & f_{1-4} & \cdots \\
f_{2-1} & f_{2-2} & f_{2-3} & \cdots & \\
f_{3-1} & f_{3-2} & f_{3-3} & f_{3-4} & \cdots \\
f_{4-1} & f_{4-2} & f_{4-3} & \cdots & \\
f_{5-1} & f_{5-2} & \cdots & & \\
\cdots & & & & \\
f_{q-1} & f_{q-2} & \cdots & f_{q-k} &
\end{array}\right]
$$

where $F_{q}$ represents one category of properties. As shown in Eq. (3), there are totally $m$ properties, which can be classified into $q$ categories.

Also, according to various travel plans, the property categories can be divided into different groups. Each group represents one category combination scheme. The classification examples are as follows:

(1) When the travel plan is "The shortest time", property categories in this group include Speed level and Congestion level:

$$
G\left(T_{1}\right)=\left\{F_{2}, F_{3}, \cdots\right\}
$$

where $G\left(T_{1}\right)$ represents the group of property categories that required to be considered under the travel plan of The shortest time $T_{1}$. Therefore when planning the route, only these two categories need to be considered.

(2) When the travel plan is "Avoiding crowded urban streets", property categories in this group include Road type and Congestion level:

$$
G\left(T_{2}\right)=\left\{F_{1}, F_{3}, \cdots\right\}
$$

(3) When the travel plan is "Avoiding the toll", property categories in this group include the Toll status:

$$
G\left(T_{3}\right)=\left\{F_{5}, \cdots\right\}
$$

(4) When the travel plan is "The least transfer", property categories in this group include Road functions:

$$
G\left(T_{4}\right)=\left\{F_{4}, \cdots\right\}
$$

In conclusion, according to different travel plans, only the corresponding property categories need to be considered when the routes are being planned, the other categories may be ignored. Property category, as the extension of the PS theory, is a new concept proposed in this paper.

In addition, the setting of the category group should take into account not only the travel plan selected by the user, but also the user's choice of vehicle. For example, when the user chooses to travel on foot, the corresponding property group should include the property of road function.

4) property combination scheme: As introduced in Section III-A3, each category has one or more properties and the categories can be divided into different groups according to the travel plan.

For example, towards one travel plan $T_{i}$, a property category combination $G\left(T_{i}\right)=\left\{F_{1}, F_{2}\right\}$ is constructed. As shown in Table II, there are 4 properties in the category $F_{1}$ (Road type) and 3 properties in the category $F_{2}$ (Speed level):

$$
\begin{aligned}
& G\left(F_{1}\right)=\left\{f_{1-1}, f_{1-2}, f_{1-3}, f_{1-4}\right\} \\
& G\left(F_{2}\right)=\left\{f_{2-1}, f_{2-2}, f_{2-3}\right\}
\end{aligned}
$$

The combination of properties in different categories can form a variety of combination schemes. As shown in Eqs. (10) and (11), category $F_{1}$ has 4 properties: National road $\left(f_{1-1}\right)$, Provincial road $\left(f_{1-2}\right)$, Country road $\left(f_{1-3}\right)$ and Street $\left(f_{1-4}\right)$; category $F_{2}$ has 3 properties: Highway $\left(f_{2-1}\right)$, Expressway $\left(f_{2-2}\right)$ and Ordinary $\left(p_{f-3}\right)$; Both can be combined to form 12 different property combination schemes, as shown in Fig. 1. 
TABLE II

ROAD PROPERTY INFORMATION TABLE

\begin{tabular}{|c|c|c|c|c|c|}
\hline Category $(F)$ & \multicolumn{5}{|c|}{ Property $(f)$} \\
\hline Road type $\left(F_{1}\right)$ & National road $\left(f_{1-1}\right)$ & Provincial road $\left(f_{1-2}\right)$ & Country road $\left(f_{1-3}\right)$ & Street $\left(f_{1-4}\right)$ & $\ldots$ \\
\hline Speed level $\left(F_{2}\right)$ & Highway $\left(f_{2-1}\right)$ & Expressway $\left(f_{2-2}\right)$ & Ordinary $\left(f_{2-3}\right)$ & $\ldots$ & \\
\hline Congestion $\left(F_{3}\right)$ & Smooth $\left(f_{3-1}\right)$ & Mild congestion $\left(f_{3-2}\right)$ & Moderate congestion $\left(f_{3-3}\right)$ & Severe congestion $\left(f_{3-4}\right)$ & $\ldots$ \\
\hline Road function $\left(F_{4}\right)$ & Only cars $\left(f_{4-1}\right)$ & Pedestrian lane $\left(f_{4-2}\right)$ & Non-motorized lane $\left(f_{4-3}\right)$ & $\ldots$ & \\
\hline Toll status $\left(F_{5}\right)$ & Toll $\left(f_{5-1}\right)$ & Toll free $\left(f_{5-2}\right)$ & & & \\
\hline$\ldots$ & & & & & \\
\hline
\end{tabular}

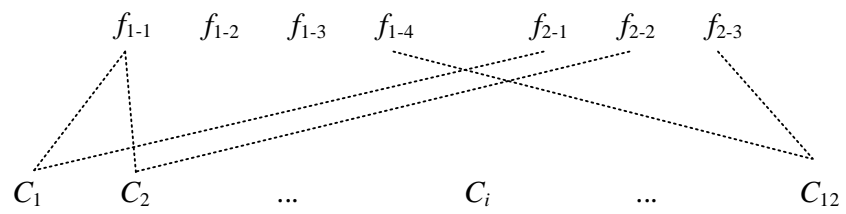

Fig. 1. Combination schemes

Each segment in the road network satisfies one of the combination schemes, where $C_{i}$ is the $i^{\text {th }}$ property combination scheme and represents one property set for some segments.

$$
F\left(a_{j}\right)=C_{1}=\left\{f_{1-1}, f_{2-1}\right\}
$$

For example, as shown in Eq. (12), $a_{j}$ represents one of those segments that have the property set $\left\{f_{1-1}, f_{2-1}\right\}$ under current travel plan $T_{i}$.

Meanwhile, scalability is an important feature of the proposed route planning system. With the change of real-time road information, the road segments' properties, as shown in Table II, can be expanded to provide more property group. In this way we can construct more property value combination schemes and satisfy more personalized multi-criterion travel preferences for users. Since the route planning can be based on combination of properties, new routing metrics can be always created and added to the system to provide services. This makes the system dynamic as well.

In addition, the real-time traffic information is collected via the mobile devices during the navigation. Then the current status of the related segment in map database can be adjusted via Message Queuing Telemetry Transport service (MQTT). MQTT has become the de facto protocol for transferring data between mobile devices and the cloud database.

\section{B. User-centric road priority setting}

Existing route planning algorithms only consider how to make the planned route shortest in the process of route planning, but ignore the user's personalized requirements for travel. Therefore, we should consider how to make the planned route satisfy the user's preferences. So, we propose to set priority for road segments according to users' different travel requirements.

As shown in Fig. 1, each combination scheme represents a different priority. When planning the route, the high-priority segments are selected first and if there are no high-priority segments, then the low-priority segments will be selected. By default, the first and second priority segments are regarded as high-priority, and the rest are low-priority segments. The details of each priority are explained below:

(1) The segments that fully meeting the requirements of travel plan (one of the property combination schemes) can be called as first-priority segments. As the user's travel preference can be quantified as multiple criteria based on the NLU (Natural Language Understanding) technology. Each criterion can be converted to a corresponding property based on the PS theory, the property combination scheme with all these properties is set as the first-priority combination. In this way the segment that with this combination is set as first-priority segment.

(2) In general, the actual traffic conditions are more complicated, and, sometimes, it is difficult to find segments that fully meet all the criteria. Considering the connectivity of the navigation road and the integrity of the route, the property combination scheme with most of these properties is set as the second-priority combination.

(3) Similarly, if there is no second-priority segment, then the requirement is lowered again and the segment that meets few properties at this stage is called third-priority segment.

The process of the priority setting is shown in Fig. 2:

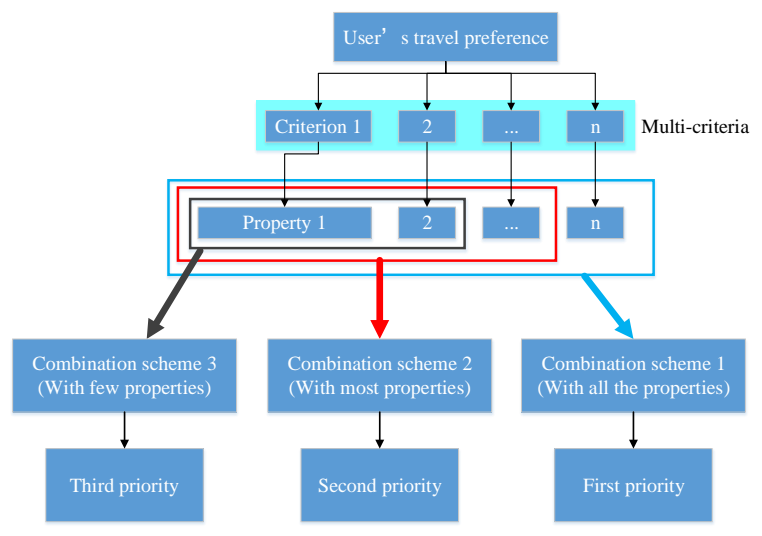

Fig. 2. Flowchart of priority setting

For example, if the travel plan is "The shortest time", the road category group corresponding to this plan includes Speed level and Congestion level. The road segments that have properties of Highway and Smooth are set as the first priority. Those having properties of Highway and Mild congestion are set as the second priority, and those having 
properties of Highway and Moderate congestion are set as third priority.

In conclusion, under different travel plans, before route planning, the system will form corresponding priority category groups. A priority represents a combination scheme of properties. After the user selects a travel plan, the system divides the priority of each property combination scheme in this travel plan and establishes the corresponding priority classification standard. According to the priority, all road segments in the road network are divided into different priority sets:

$$
\begin{aligned}
& G\left(C_{1}\right)=\left\{a_{1}, \cdots, a_{i}\right\} \\
& G\left(C_{2}\right)=\left\{a_{i+1}, \cdots, a_{i+j}\right\} \\
& \cdots \\
& G\left(C_{12}\right)=\left\{a_{i+j+\cdots+1}, \cdots, a_{p}\right\}
\end{aligned}
$$

where $G$ represents the group of segments.

\section{Route planning process}

In the process of route planning, the widely implemented algorithms, such as Dijkstra, traverse all the segments connected with the current vertex. Although this approach can guarantee to find the shortest path, it requires a large amount of computation and is less efficient. Therefore, this paper proposed an optimized progressive route planning process.

Based on the PS-based road property description and the setting of priority in section III-A and III-B, we proposed an efficient route planning process. This process includes 8 steps, mainly comprised of building the map database, segment selection and starting navigation stages.

(1) Building map database Step1: Pre-built map database, comprised of two parts:

One is the road property database: it comprises of the property information of each road segment, such as congestion status (smooth, mild congestion, moderate congestion, severe congestion), road types (highway, expressway, ordinary road), length of the road segment, road speed limit, etc. For a segment, the property it owns is marked as 1; otherwise, it is marked as 0 .

Another is the priority database of road segment selection. For different navigation choices, road segment has different priority classification criteria. Each segment is assigned with different priority according to the corresponding division criteria. First-priority is the first, followed by the secondpriority, third-priority, and so on.

(2) Segment selection

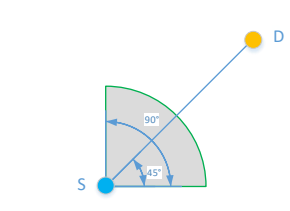

(a) Traversal segments in $90^{\circ}$

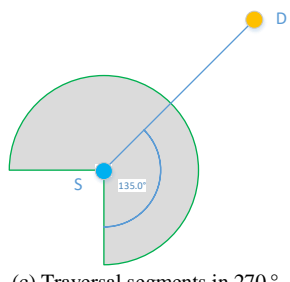

(c) Traversal segments in $270^{\circ}$

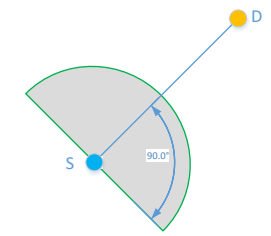

(b) Traversal segments in $180^{\circ}$

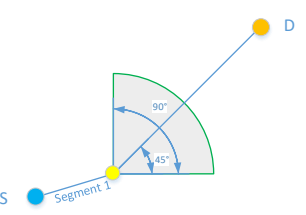

(d) Look for segment 2
Fig. 3. Traverse all the road segments

Step2: A navigation path will include multiple segments. First, an empty set of segments will be established. The optimal path is formed by connecting segments end-to-end.

Step3: Based on the connection between starting point and destination, we traverse all the road segments connected to the starting point in a $90^{\circ}$ area, which is shown in Fig .3(a). By default, the first and second priority segments are highpriority segments, and the rest are low-priority segments.

Step4: When more than one road segment is connected with the starting point, according to the travel plan set by the user, the road segment meeting the first priority will be selected. If there is no road segment meeting the first priority, then the road segment meeting the second priority will be selected.

Step5: If the road segment meeting the requirements (user's preferences) cannot be found within the $90^{\circ}$ area, then the search range is expanded to $180^{\circ}$ and Step 4 is performed, where the highest priority road segment is selected in this area, which is shown in Fig. 3(b). Also, if the road segment meeting the requirement is still not found, the search range is further expanded to $270^{\circ}$ and repeat Step 4, which is shown in Fig. 3(c). If still no road segment can't be found to meet the requirements, it will narrow down the search range to the original $90^{\circ}$ area and lower the priority again until a road segment is selected in this area. Then the selected segment is added to the segment set in this navigation. If the road segment meeting the requirements is not found at last, the user is prompted to have no planned route, please re-select.

Step6: Take the end point of the selected segment as the new starting point, perform Steps 3-5 again, and start to select the next segment until the destination set by the user is reached, which is shown in Fig. 3(d).

Step7: At the same time, after each selection of a road segment, a loop avoidance mechanism will be ignited to avoid falling into an endless loop:

As shown in Fig.4(a), when the segment $d$ is connected to the route, $e$ is the best segment to be connected. Therefore, segment $e$ is chosen into the segment set. Then the segment $f$ and segment $g$ are chosen into the segment set. There 
forms a loop as $d \rightarrow e \rightarrow f \rightarrow g \rightarrow d$. In the loop avoiding mechanism, when a road segment is selected, it should check the route segment set to find whether this segment is already in the set or not. If this segment is already in the set, it means that a loop has been created. So, the next segment is labelled as a loop segment and the route information on this segment is to be deleted. Then another segment is to be found. As shown in Fig.4(b), when a route $d \rightarrow e \rightarrow f \rightarrow g \rightarrow d$ has formed a loop, segment $e$ is labelled and the route $d \rightarrow e$ is deleted. Then perform Step 6 to choose another segment, segment $h$ is to be found. At last a final route is discovered and shown in Fig.4(c).

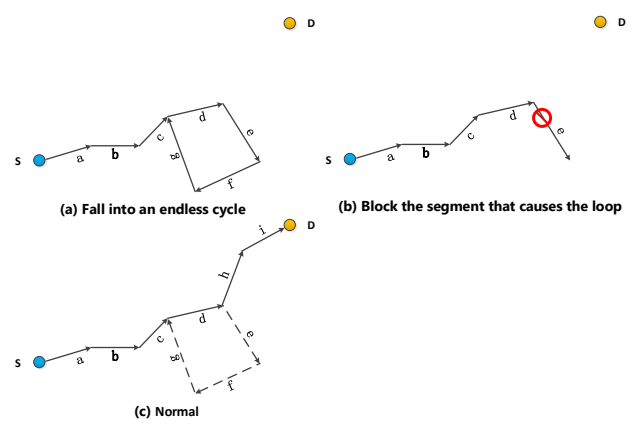

Fig. 4. Loop avoidance strategy

(3) Starting navigation

Step8: All road segments saved in the segment set are connected end to end, which is the route planned for this navigation. After planning the route, start providing navigation services for users on their mobile terminal. In the navigation process, the system can obtain the realtime traffic information via the mobile crowdsensing manner anytime. The collected information could be uploaded to the background database via Message Queue service (MQ) and the priority of segments in database could be adjusted.

The pseudocode of user-centric route planning algorithm based on PS theory is shown in Algorithm 1:

As shown in algorithm 1, in this case, the time complexity of Function while is $O(n)$, so the overall time complexity is:

$$
T(n)=O\left(n^{2}\right)
$$

The space complexity is:

$$
S(n)=O(1)
$$

\section{Algorithm Implementation AND EVAluation}

\section{A. Experimental Setup}

1) Evaluation environment and target: In order to model and implement the proposed algorithm, experiments were conducted by using MATLAB R2016a on a PC with a 64bit Windows 10 operating system, a 16 GB of RAM, and a 1.19-GHz-Core(TM) i5-based processor. The source code of the experiment has been published on Github platform [23].

In order to verify the effectiveness of our proposed usercentric and multi-criterion route planning algorithm, we have

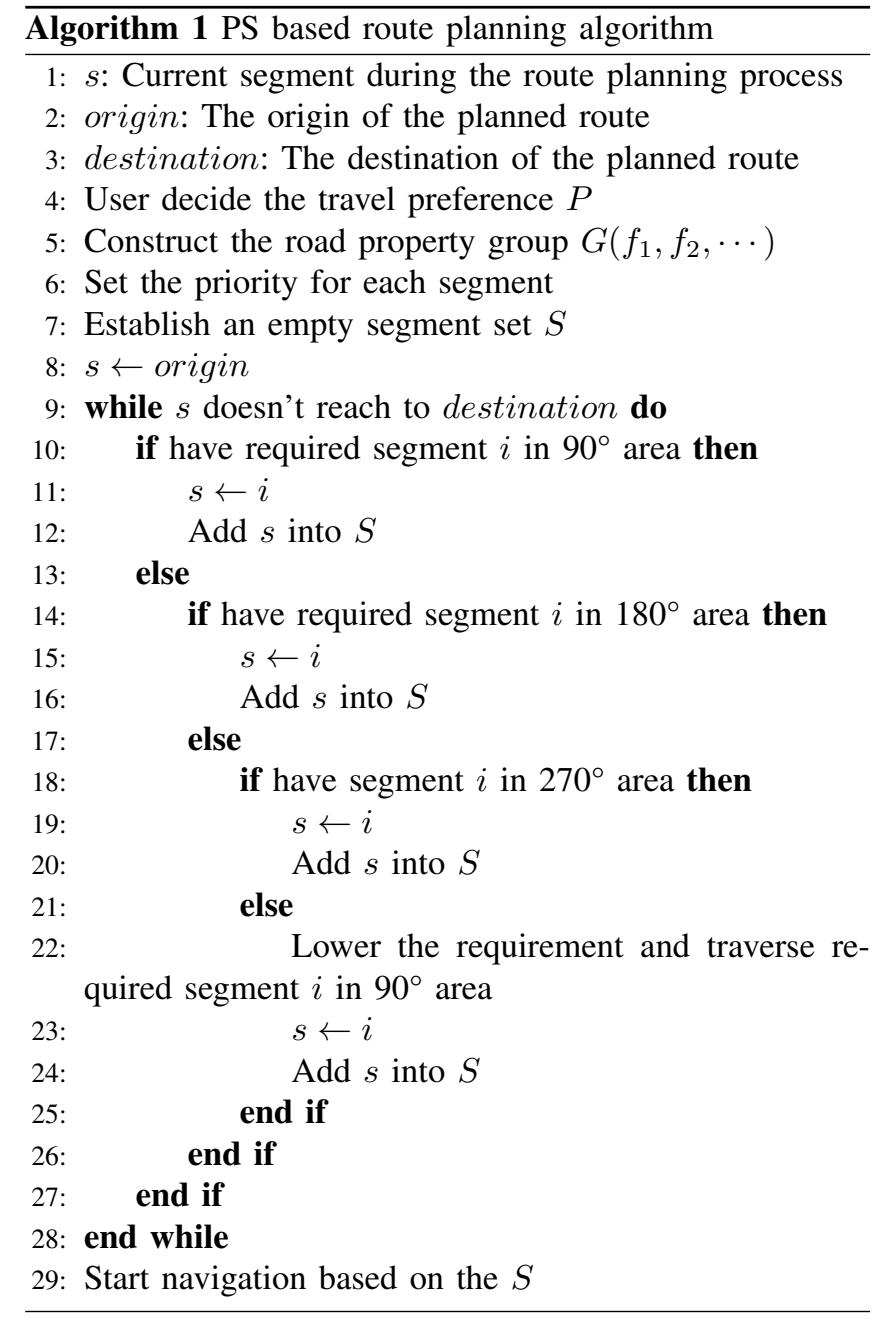

compared with the following two widely used route planning algorithms, Greedy Forwarding geographical Routing (GRF) and the improved Dijkstra algorithm on Graphhopper.

(1) GFR is carried out by default in a greedy way: The current node always forwards the message to the neighbor node closest to the target [24]. A simple and effective iterated greedy algorithm is presented in [25].

(2) Graphhopper is an open source routing library and server written in Java and provides a web interface called GraphHopper Maps, which has been widely used [26]. Graphhopper can be configured to use different algorithms, such as Dijkstra, $A^{*}$, and its bidirectional versions. In order to make the routing fast enough for long paths (continental size) and avoid heuristic approaches, Graphhopper uses contraction hierarchies by default.

The Graphhopper routing service based on Dijkstra algorithm was used for the comparison experiments in this paper. [27] proposed a multi-criterion decision making of optimal route based on Dijkstra algorithm.

2) Data Preparation: In our experiments, the proposed algorithm is tested and validated through a case study of a real driving scenario in London, the UK. We take the road network in an urban area in London as an example, where 
the actual road network is shown in Fig.5:

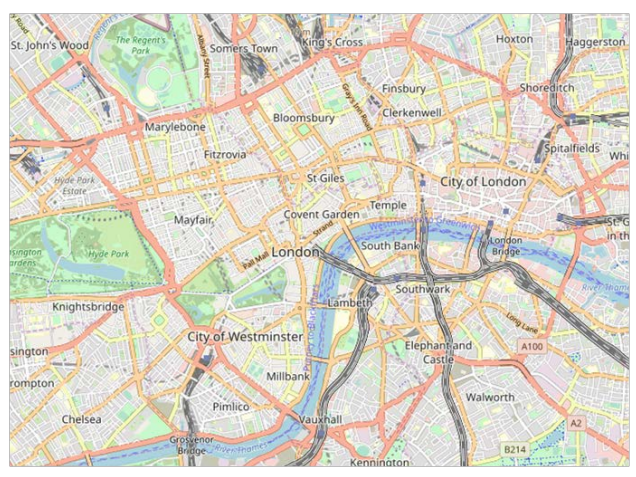

Fig. 5. A regional road network

In order to compare the performance of proposed algorithm with the planned route on Graphhopper, we simplify and show the main roads of actual city road network using Matlab, which is shown in Fig.6:

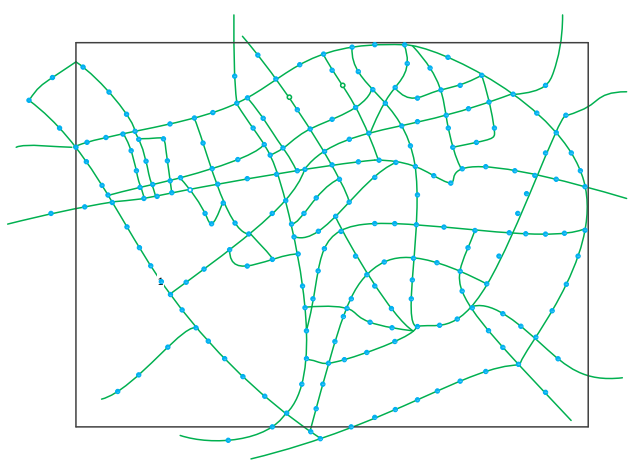

Fig. 6. Linearization of regional roads

There are totally 216 road segments in this area. The map database in this experiment is the 216 segments with their properties.

3) Evaluation Metrics: Degree of Demand Satisfaction (DDS) is the major metric used to evaluate the proposed algorithm. In addition, Time and Distance are also two important evaluation metrics to evaluate the performance.

(1) DDS is one of the the evaluation indices used to evaluate that, to what extend, the planned route meets the travel plan set by the user, which is defined as:

$$
D D S=\frac{S_{f}+S_{c}}{S_{T}}
$$

where $S_{f}$ represents the first-priority segments in the planned route, $S_{c}$ represents the second-priority segments in the planned route, both are combined as the high-priority segments which highly meeting the travel plan. $S_{T}$ represents the total distance of the planned route.

(2) The shortest time and the shortest distance are always the users' default travel preferences. In this case, we need to provide users with a planned route as short as possible in terms of time and distance while ensuring users' DDS.

\section{B. Experimental steps and computation}

1) Experimental steps: First, the starting point, destination point and user's travel plan are set. These parameters are the input of the experiment.

Second, the system constructs the segment property group and the corresponding priority setting according to the travel plan.

Finally, plan the route from the starting point based on the proposed algorithm, Dijkstra and GFR methods, respectively.

2) The computation of metrics: First of all, we need to calculate the DDS. The calculation method is shown in Eq. (16).

For the calculation of travel distance, the distance of each road section in the database is consistent with the real-world actual distance.

In order to calculate the travel time conveniently, we set the speed of the road segment reasonably according to different road type and different congestion level. Each type of road, such as city road or street, has its usual speed limit. The speed of different segments is set in a hierarchical way. Specific calculation method is shown in Eq. (17):

$$
T=\frac{d_{1}}{s_{1}}+\frac{d_{2}}{s_{2}}+\cdots+\frac{d_{n}}{s_{n}}
$$

where $T$ represents the travel time, $d_{n}$ represents the distance of the $n^{t h}$ segment and $s_{n}$ represents the speed limit of the $n^{\text {th }}$ segment.

\section{Performance Evaluation}

1) Comparison of travel plan "Smooth highway first": First of all, when the user's travel plan is "smooth highway first", the road properties that need to be considered are road congestion and road type. The road property diagram is shown in Fig.7, $S$ represents the starting point and $D$ represents the destination.

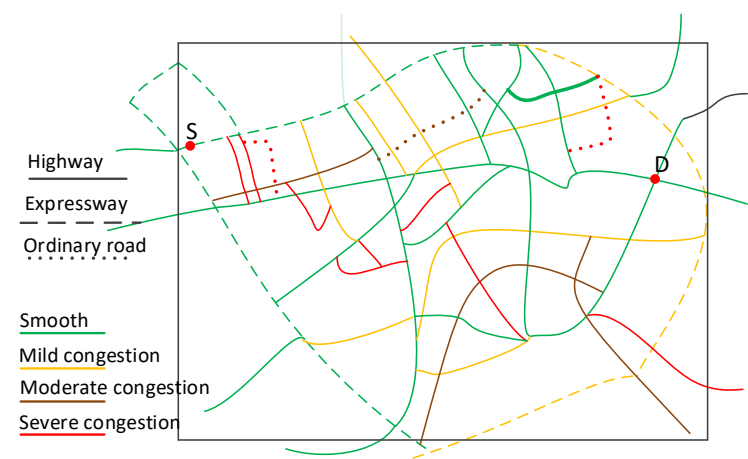

Fig. 7. Road feature (plan 1)

Road priorities are set in Table III.

The route planned by Graphhopper using Dijkstra algorithm is shown in Fig.8. 
TABLE III

SEGMENT PRIORITY SETTING (PLAN 1)

\begin{tabular}{|c|c|c|c|c|c|c|c|}
\hline Smooth & Mild congestion & Moderate Congestion & Severe congestion & Highway & Expressway & Ordinary road & Priority \\
\hline 1 & 0 & 0 & 0 & 1 & 0 & 0 & $\mathbf{1}$ \\
\hline 0 & 1 & 0 & 0 & 1 & 0 & 0 & 2 \\
\hline 0 & 0 & 1 & 0 & 1 & 0 & 0 & 3 \\
\hline 0 & 0 & 0 & 1 & 1 & 0 & 0 & 4 \\
\hline 1 & 0 & 0 & 0 & 0 & 1 & 0 & 5 \\
\hline 0 & 1 & 0 & 0 & 0 & 1 & 0 & 6 \\
\hline 0 & 0 & 1 & 0 & 0 & 1 & 0 & 7 \\
\hline 0 & 0 & 0 & 1 & 0 & 1 & 0 & 8 \\
\hline 1 & 0 & 0 & 0 & 0 & 0 & 1 & 9 \\
\hline 0 & 1 & 0 & 0 & 0 & 0 & 1 & 10 \\
\hline 0 & 0 & 1 & 0 & 0 & 0 & 1 & 11 \\
\hline 0 & 0 & 0 & 1 & 0 & 0 & 1 & 12 \\
\hline
\end{tabular}

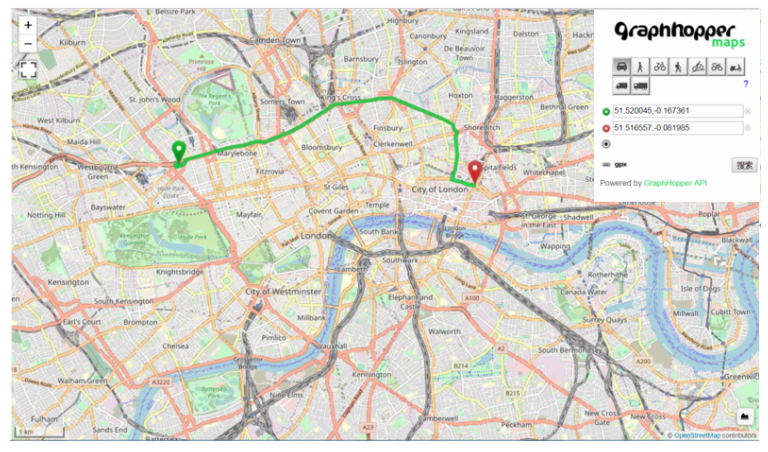

Fig. 8. Planned route in Graphhopper (plan 1)

The planned routes based on PS theory, GFR algorithm, and Dijkstra algorithm are shown in Fig.9, which are demonstrated by bold lines. Among them, as shown in Fig.9, the planned route based on Dijkstra algorithm is the simplified.

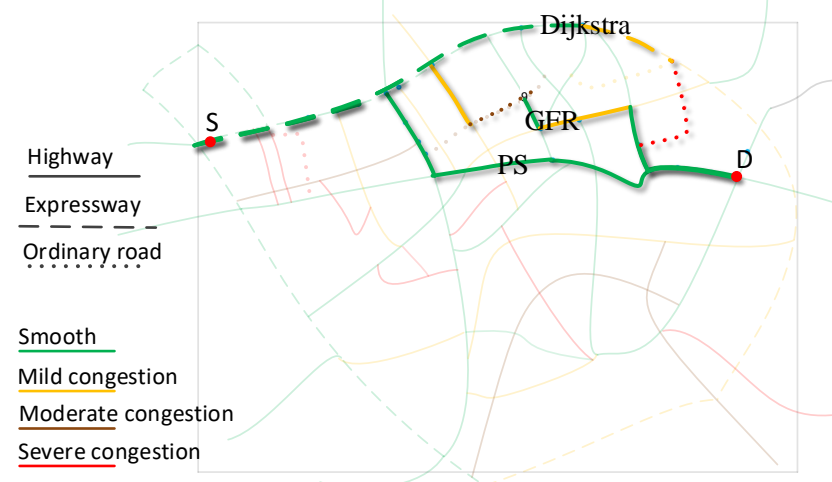

Fig. 9. Planned routes based on PS, GFR, and Dijkstra algorithm (plan 1)

The comparison of the three planned routes is shown in Fig. 10. It can be seen from Fig. 10, because the route planning based on PS algorithm takes the requirements of users on road type and road congestion into account, the recommended route achieved a much higher degree of user's demand satisfaction than the other two algorithms. In addition, although there is little difference in the distance of the planned route obtained by the three algorithms, the planned route based on PS algorithm is mainly composed of smooth segments, so the whole journey time of the obtained road is shorter.

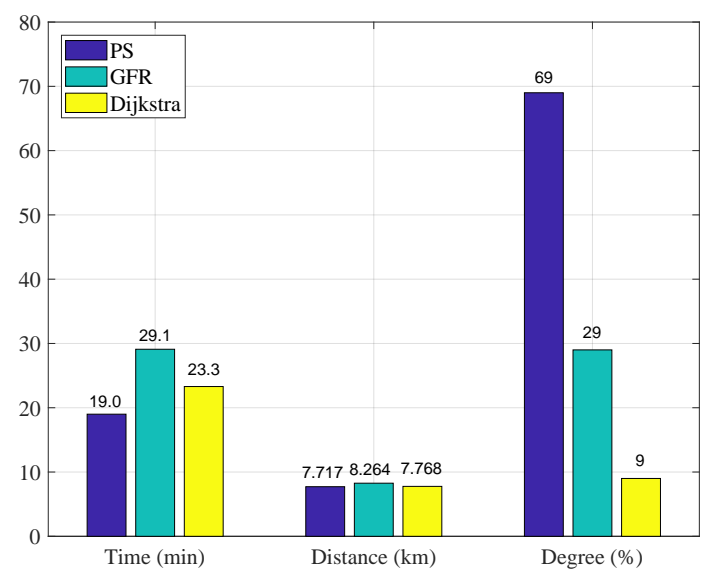

Fig. 10. Comparison of three algorithms (plan 1)

The proportion of different types of segments included in the three planned routes lead to the difference in user's satisfaction. As can be seen from Fig. 9, the planned route based on the PS algorithm proposed in this paper is mainly composed of smooth highway. Only when there is no highway at the starting point, the system will choose the smooth expressway. While, the planned route based on the GFR algorithm and Dijkstra algorithm does not consider the requirements of users, so the planned travel route includes ordinary roads and congested segments.

2) Comparison of travel plan "Avoid the speed limited road": Then, we test the second plan. When the user's travel plan is - "avoid speed limited road", we need to consider whether the road segment is speed limit or not. The road property diagram is shown in Fig. 11, where $S$ represents the starting point and the $D$ represents the destination. 


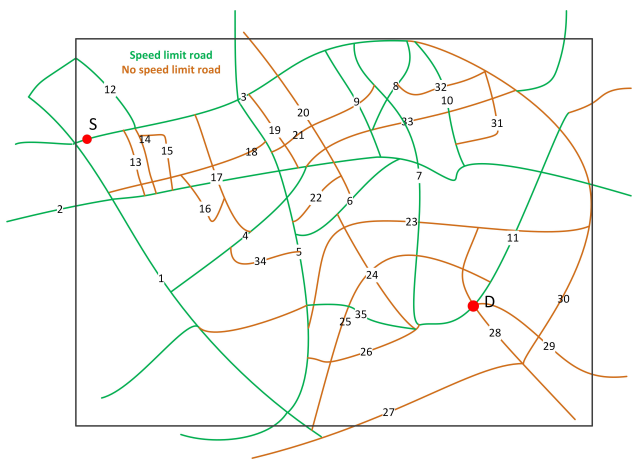

Fig. 11. Road feature (plan 2)

Road priorities are set as Table IV:

TABLE IV

SEGMENT PRIORITY SETTING (PLAN 2)

\begin{tabular}{|c|c|c|}
\hline Speed limit & No speed limit & Priority \\
\hline 0 & 1 & $\mathbf{1}$ \\
\hline 1 & 0 & $\mathbf{2}$ \\
\hline
\end{tabular}

The route planned by Graphhopper using Dijkstra algorithm is shown in the Fig.12:

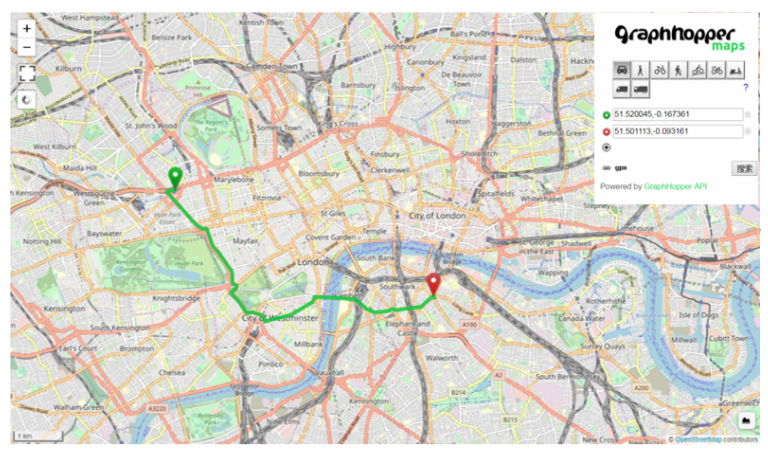

Fig. 12. Planned route in Graphhopper (plan 2)

The planned routes based on PS theory, GFR algorithm, and Dijkstra algorithm are shown in the Fig. 13, which are demonstrated by dotted lines for the route designed by PS, solid lines for GFR algorithm and dashed lines for Dijkstra, respectively. The planned route based on Dijkstra algorithm is the simplified result of the route planned by Graphhopper.

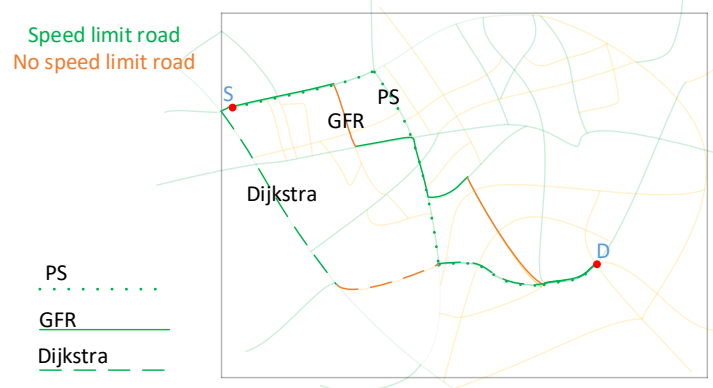

Fig. 13. Planned routes based on PS, GFR, and Dijkstra algorithm (plan 2)

The performance of the planned routes obtained by the three algorithms is shown in Fig. 14. As can be seen from Fig. 14, the planned route based on PS algorithm does not include the speed limit segment, so the degree of user's demand satisfaction has reached $100 \%$. At the same time, although the consideration of road speed limit is added in the route planning, the planned route based on PS algorithm does not significantly increase the road distance, and the travel time is shorter than the other two routes.

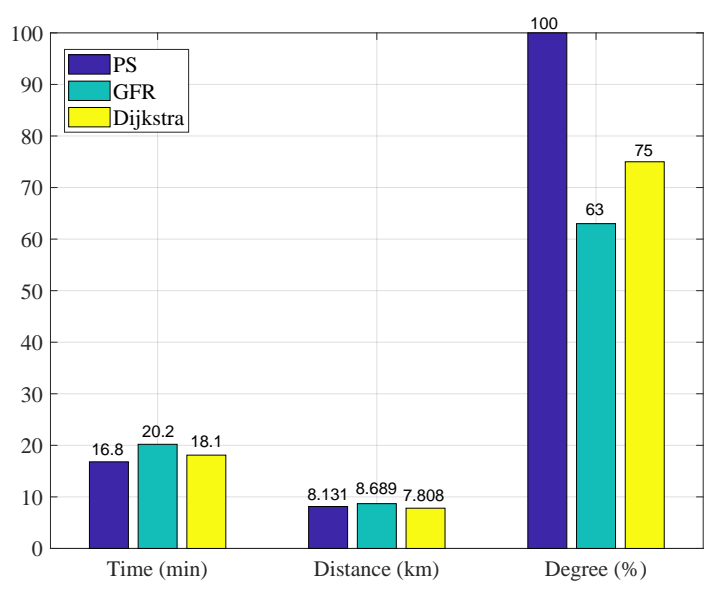

Fig. 14. Comparison of three algorithms (plan 2)

The type of segments included in the three planned routes leads to the difference in user's satisfaction. As can be seen from Fig. 13, considering the requirement of "avoid the speed limit road", the route planned based on PS algorithm can avoid the speed limit segment very well. However, the other two algorithms do not consider the road property of "speed limit or not" in the route planning, so the planned route contains the speed-limited segments, which will bring a negative impact on user's travel experience.

\section{CONCLUSiON}

In this paper, a user-centric route planning algorithm is proposed. Based on the PS theory, this algorithm focuses on considering the multi-property information of the road when carrying out route planning, and provides diversified travel 
routes for users according to the personalized differences on travel demand. At the same time, the system can update the road property database in real-time according to the feedback of users, and then provide users with more accurate route planning services. Experimental results show that the planned route based on PS algorithm not only meets the requirements of users, but also is very close to the shortest route between two points.

In the future, we will consider more properties to design and optimize the routing algorithm, so as to make better travel planning under the modern complex road network.

\section{REFERENCES}

[1] H. Bast, D. Delling, A. Goldberg, M. Müller-Hannemann, T. Pajor, P. Sanders, D. Wagner, and R. F. Werneck, "Route planning in transportation networks," in Algorithm engineering. Springer, 2016, pp. $19-80$.

[2] Z. Wen, L. Kong, G. Chen, M. K. Khan, S. Mumtaz, and J. J. Rodrigues, "Understanding multi-path routing algorithms in datacenter networks," in 2019 IEEE Global Communications Conference (GLOBECOM). IEEE, 2019, pp. 1-6.

[3] S. Liao, J. Wu, J. Li, A. K. Bashir, S. Mumtaz, A. Jolfaei, and N. Kvedaraite, "Cognitive popularity based ai service sharing for software-defined information-centric networks," IEEE Transactions on Network Science and Engineering, vol. 7, no. 4, pp. 2126-2136, 2020.

[4] Y. Ma, Y. Yuan, G. Wang, X. Bi, and H. Qin, "Trust-aware personalized route query using extreme learning machine in location-based social networks," Cognitive Computation, vol. 10, no. 6, pp. 965-979, 2018.

[5] D. Papinski and D. M. Scott, "A gis-based toolkit for route choice analysis," Journal of Transport Geography, vol. 19, no. 3, pp. 434442, 2011.

[6] T. Mátrai, J. Tóth, and M. T. Horváth, "Route planning based on urban mobility management," Hungarian Journal of Industry and Chemistry, pp. 71-79, 2016.

[7] P. Pahlavani and M. R. Delavar, "Multi-criteria route planning based on a driver's preferences in multi-criteria route selection," Transportation research part C: emerging technologies, vol. 40, pp. 14-35, 2014.

[8] J. Dibbelt, C. Konstantopoulos, D. Wagner, D. Gavalas, S. Kontogiannis, C. Zaroliagis, V. Kasapakis, and G. Pantziou, "Multimodal route and tour planning in urban environments," in 2017 IEEE Symposium on Computers and Communications (ISCC). IEEE, 2017, pp. 214219.

[9] E. W. Dijkstra et al., "A note on two problems in connexion with graphs," Numerische mathematik, vol. 1, no. 1, pp. 269-271, 1959.

[10] D. Delling, A. V. Goldberg, T. Pajor, and R. F. Werneck, "Customizable route planning," in International Symposium on Experimental Algorithms. Springer, 2011, pp. 376-387.

[11] Y. Zhou, J.-C. Thill, and Z. Huang, "Design of a user-centric decision support tool for fixed-route bus travel planning," Applied Geography, vol. 31 , no. 3, pp. 1173-1184, 2011.

[12] S. Funke and S. Storandt, "Personalized route planning in road networks," in Proceedings of the 23rd SIGSPATIAL International Conference on Advances in Geographic Information Systems, 2015, pp. $1-10$

[13] G. Cui, J. Luo, and X. Wang, "Personalized travel route recommendation using collaborative filtering based on gps trajectories," International journal of digital earth, vol. 11, no. 3, pp. 284-307, 2018.

[14] B. Yang, C. Guo, Y. Ma, and C. S. Jensen, "Toward personalized, context-aware routing," The VLDB Journal, vol. 24, no. 2, pp. 297$318,2015$.

[15] S. Nadi and M. R. Delavar, "Multi-criteria, personalized route planning using quantifier-guided ordered weighted averaging operators," International Journal of Applied Earth Observation and Geoinformation, vol. 13, no. 3, pp. 322-335, 2011.

[16] A. S. Niaraki and K. Kim, "Ontology based personalized route planning system using a multi-criteria decision making approach," Expert Systems with Applications, vol. 36, no. 2, pp. 2250-2259, 2009.
[17] M. Torres, D. A. Pelta, and J. L. Verdegay, "Proa: an intelligent multi-criteria personalized route assistant," Engineering Applications of Artificial Intelligence, vol. 72, pp. 162-169, 2018.

[18] A. Abdelrahman, A. S. El-Wakeel, A. Noureldin, and H. S. Hassanein, "Crowdsensing-based personalized dynamic route planning for smart vehicles," IEEE Network, vol. 34, no. 3, pp. 216-223, 2020.

[19] C. Chen, D. Zhang, B. Guo, X. Ma, G. Pan, and Z. Wu, "Tripplanner: Personalized trip planning leveraging heterogeneous crowdsourced digital footprints," IEEE Transactions on Intelligent Transportation Systems, vol. 16, no. 3, pp. 1259-1273, 2014.

[20] M. Baum, J. Dibbelt, T. Pajor, and D. Wagner, "Dynamic timedependent route planning in road networks with user preferences," in International Symposium on Experimental Algorithms. Springer, 2016, pp. 33-49.

[21] Y. Ding, C. Chen, S. Zhang, B. Guo, Z. Yu, and Y. Wang, "Greenplanner: Planning personalized fuel-efficient driving routes using multisourced urban data," in 2017 IEEE International Conference on Pervasive Computing and Communications (PerCom). IEEE, 2017, pp. 207-216.

[22] X. Wang and S. Li, "Scalable routing modeling for wireless ad hoc networks by using polychromatic sets," IEEE Systems Journal, vol. 7, no. 1 , pp. 50-58, 2012.

[23] P. Li, "Polychromatic-sets-theory," https://github.com/peisong0109/ Polychromatic-Sets-Theory.

[24] J. Na, D. Soroker, and C.-k. Kim, "Greedy geographic routing using dynamic potential field for wireless ad hoc networks," IEEE Communications Letters, vol. 11, no. 3, pp. 243-245, 2007.

[25] X. Wang, S. Wang, L. Wang, H. Zheng, J. Hao, R. He, and Z. Sun, "An effective iterated greedy algorithm for online route planning problem," in 2020 IEEE Congress on Evolutionary Computation (CEC). IEEE, 2020, pp. 1-8.

[26] V. Nallur, A. Elgammal, and S. Clarke, "Smart route planning using open data and participatory sensing," in IFIP International Conference on Open Source Systems. Springer, 2015, pp. 91-100.

[27] Y. D. Rosita, E. E. Rosyida, and M. A. Rudiyanto, "Implementation of dijkstra algorithm and multi-criteria decision-making for optimal route distribution," Procedia Computer Science, vol. 161, pp. 378$385,2019$.

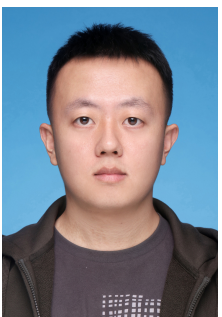

Peisong $\mathbf{L i}$ is currently a $\mathrm{PhD}$ student in School of Advanced Technology at Xi'an JiaotongLiverpool University, Suzhou, China. He received the B.E. degree in Optoelectronic Information Science and Engineering from Guilin University of Electronic and Technology, Guilin, China in 2017 and the M.Sc. degree in Communication and Information System from Shanghai Maritime University, Shanghai, China in 2020, respectively. 


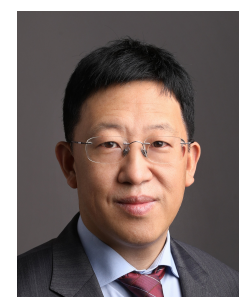

Xinheng Wang (M'04 -SM'14) received the B.E. and M.Sc. degrees in electrical engineering from Xian Jiaotong University, Xian, China, in 1991 and 1994, respectively, and the Ph.D. degree in electronics and computer engineering from Brunel University, Uxbridge, U.K., in 2001. He is currently a Professor with the School of Advanced Technology and acting the Head of Department of Mechatronics and Robotics, Xi'an JiaotongLiverpool University (XJTLU), Suzhou 215123, China. Prior to joining XJTLU, he was a professor with different universities in the UK. He is a general chair/technical programme chair for CollaborateCom over the last five years and a guest editor for a number of international journals.

$\mathrm{He}$ has been an Investigator or Co-Investigator of nearly 30 research projects sponsored from EU, UK EPSRC, Innovate UK, China NSFC, and industry. The research has led to the publications of over 180 referred papers, 15 granted patents, and development of a few commercial products in condition monitoring, wireless mesh networks, and user-centric routing and navigation for group users. The smart trolley he has developed with an industry partner is the first airport Internet of Things (IoT) product in the world to provide smart services to air passengers. His current research interests lie in industrial IoT, edge AI for industrial machine vision, indoor positioning, acoustic localization, communications and sensing, and personalized navigation and services for group travelers.

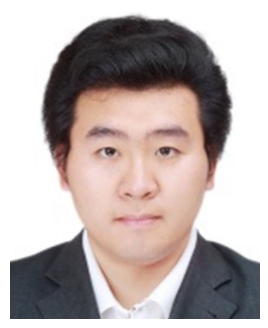

Honghao Gao received a Ph.D. in Computer Science and started his academic career at Shanghai University in 2012. Prof. Gao is currently with the School of Computer Engineering and Science, Shanghai University, China. He is also a professor at Gachon University, South Korea. Prior to that, he was a research fellow at the Software Engineering Information Technology Institute at Central Michigan University (CMU), USA and was an adjunct professor at Hangzhou Dianzi University, China. His research interests include formal software verification, industrial IoT/wireless networks, service collaborative computing, and intelligent medical image processing. He has publications in IEEE TII, IEEE T-ITS, IEEE IoT-J, IEEE TNSE, IEEE TCCN, IEEE TGCN, IEEE/ACM TCBB, ACM TOIT, ACM TOMM, ACM TMIS, IEEE TCSS, IEEE TETCI, IEEE JBHI, and IEEE Network. Prof. Gao is a fellow of IET, BCS, and EAI and a senior member of IEEE, CCF, and CAAI. He is the Editor-in-Chief of the International Journal of Intelligent Internet of Things Computing (IJITTC), Editor of Wireless Network (WINE) and IET Wireless Sensor Systems (IET WSS), and Associate Editor for IEEE Transactions on Intelligent Transportation Systems(IEEE T-ITS), IET Software, International Journal of Communication Systems(IJCS), and Journal of Internet Technology(JIT). Moreover, he has broad working experience in cooperative industry-university research. He is a European Union Institutions-appointed external expert for reviewing and monitoring the EU Project, is a member of the EPSRC Peer Review Associate College for UK Research and Innovation in the UK, and is also a founding member of the IEEE Computer Society Smart Manufacturing Standards Committee.

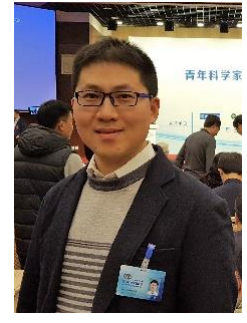

Xiaolong Xu is currently a professor in the School of Computer Science, Nanjing University of Posts \& Telecommunications, Nanjing, China. He is also working for the Jiangsu Key Laboratory of Big Data Security \& Intelligent Processing. He received the B.E. in computer and its applications, M.E. in computer software and theories and Ph.D. degree in communications and information systems, in 1999, 2002 and 2008, respectively.

$\mathrm{He}$ is a senior member of China Computer Federation. He teaches graduate courses and conducts research in areas of Cloud Computing, Big Data, Information Security and Novel Network Computing Technologies.

As the leader of project teams, he has successfully completed a number of high-level research projects, including the projects sponsored by the National Science Fund of China. He has published more than 100 Journal and conference papers as the first or corresponding author and 5 books. He is authorized 52 patents by the State Intellectual Property Office of China as the first inventor. He was rated as excellent young professor of Jiangsu Province in 2014, selected as the high-level creative talents of Jiangsu province in 2015, and won the title of outstanding expert in the area of computer science and technology.

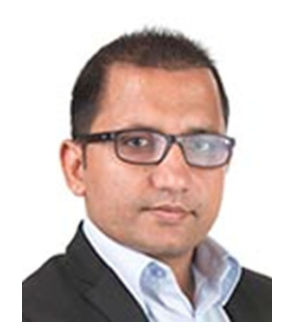

Muddesar Iqbal Dr Iqbal won an EPSRC Doctoral Training Award in 2007 and completed his $\mathrm{PhD}$ from Kingston University in 2010 in Wireless Networks. He has been a principal investigator, co-investigator, project manager, coordinator and focal person of more than 15 internationally teamed research and development, capacity building and training projects. In September 2008, he acquired the Status of Foreign Expert from the State Administration of Foreign Experts Affairs, People's Republic of China. He is member of British Computer Society and also a British Council's Active Citizen for the implementation of British Council Social Action Projects under Youth, Education and Society. He is serving as aMember Board of Governor for United Nation's Independent Monitoring Association for Child Labour (IMAC), which was set up by the International Labour Organisation's International Programme on the Elimination of Child Labour (IPEC) from the Sailkot's soccer ball industry in Pakistan.

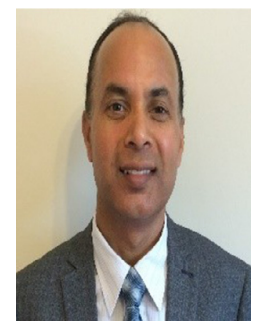

Keshav Dahal is a Professor of Intelligent Systems and the leader of the Artificial Intelligence, Visual Communication and Network (AVCN) Research Centre at the University of the West of Scotland (UWS), UK. He is also affiliated with Nanjing University of Information Science and Technology (NUIST) China. Before joining UWS he was with Bradford and Strathclyde Universities in UK. He obtained his Ph.D. and Master degrees from the University of Strathclyde, UK. His research interests lie in the areas of applied AI to intelligent systems, trust and security modeling in distributed systems, and scheduling/optimization problems. He has published extensively with award winning papers, and has sat on organizing/program committees of over 60 international conferences including as the General Chair and Programme Chair. He is a senior member of the IEEE. 